\title{
Trauma Surgery $\&$ Acute Care Open \\ Quantitative analysis of intermuscular septa in the leg: implications for trauma surgery
}

\author{
Lorreen Agandi (D) ,' Kristina Fuller, ${ }^{1}$ Kristin Sonderman, ${ }^{2}$ Samuel Tisherman (D) , ${ }^{3}$ \\ Adam C Puche 4
}

'Shock Trauma and

Anesthesiology Research, R Adams Cowley Shock Trauma Center, Baltimore, Maryland, USA

${ }^{2}$ Shock Trauma Center, University of Maryland Medical Center, Baltimore, Maryland, USA ${ }^{3}$ Surgery and the Program in Trauma, University of Maryland School of Medicine, Baltimore, Maryland, USA

${ }^{4}$ Anatomy and Neurobiology, University of Maryland School of Medicine, Baltimore, Maryland, USA

Correspondence to DrAdam C Puche; apuche@ som.umaryland.edu

Received 18 February 2021 Accepted 13 June 2021
ABSTRACT

Background Compartment syndrome is the excess swelling within an inelastic compartment leading to excessive compartment pressure. Lower limb trauma has a high risk of compartment syndrome, which is typically mitigated using a two-incision fasciotomy. Our previous findings showed surgeons sometimes perform incomplete fasciotomies due to misidentifying the septum between the lateral and superficial posterior compartments as the septum between the anterior and lateral compartments. We conjectured this may be due to variability in the septal position between individuals leading to misinterpretation of the septal identity. Methods A retrospective analysis was performed using $\mathrm{CT}$ angiograms to analyze septal position between the anterior and lateral compartments of the leg of 100 patients randomly selected from the University of Maryland Shock Trauma Center database.

Results Analysis of septal position showed that (1) as the septum progresses distally down the leg, the relative septum position shifts anteriorly; and that (2) there was considerable variability in the intermuscular septum position between individuals even when accounting for the anterior to posterior progression of septal position. Discussion This variability could lead to erroneous septal identification in individuals with a very anteriorly located septum during a leg fasciotomy with the classic initial incision being insufficiently anterior. We propose making the lateral initial incision 'two finger breadths posterior the tibia' rather than the traditional 'one finger breadth anterior' to the fibula. This moves the initial incision slightly anteriorly, uses the more readily palpable tibia, and makes the medial and lateral incisions symmetrical at 'two finger breadths' from the tibia, simplifying the procedure.

Level of evidence Level 3.

\section{BACKGROUND}

Compartment syndrome is characterized as excess swelling within the compartment leading to (or due to) an increase in pressure in a limited space. ${ }^{1}$ When compartment pressures exceed vascular inflow, tissue ischemia occurs. If not treated, it can cause tissue necrosis, functional impairment, potential need for amputation and risk of death. The window of effective surgical intervention is $3-5$ hours after injury. $^{2}$

The leg is treated more often than other areas for compartment syndrome. A two-incision lower extremity fasciotomy is commonly performed to mitigate the syndrome in trauma surgery. ${ }^{3}$ Surgeons are taught the technique of placing the medial incision two finger breadths posterior to the tibia and the lateral incision two finger breadths anterior to the fibula. ${ }^{3}$ Surgeons who take the Advanced Surgical Skills for Exposure in Trauma (ASSET) course are taught to place the medial incision one thumb or two finger breadths posterior to the tibia and the lateral incision one finger breadth in front of the fibula. ${ }^{4}$ Thus, there is disagreement in the literature regarding incision landmarks and positioning.

Our previous study of ASSET-trained surgeons performing procedures on cadavers demonstrated that compartments, particularly anterior and deep posterior, were frequently missed. ${ }^{5}$ We hypothesized that anterior compartment error rate may be due to incision position relative to intermuscular septum position between the anterior and lateral compartments. Previous studies looking at variability in the leg only assessed compartment pressures ${ }^{6}$ and not positioning of leg compartments. We conjectured that using the fibular landmark places the incision too posterior relative to the intermuscular septum such that the surgeon proceeds posteriorly, finding the septum between lateral and superficial posterior compartments rather than the septum between anterior and lateral compartments, consequently failing to decompress the anterior compartment.

The goal of this study was to investigate variability in the position of the intermuscular septum relative to the fixed bony landmarks of the tibia and fibula and if there are any patient demographics (height, body habitus and muscle mass) that may be predictive of septal position. We further hypothesized that leg injury could result in edema or disruption that shifts the septal position when compared with injuries to another part of the lower extremity (eg, thigh/foot injury).

\section{METHODS}

A retrospective analysis was performed using measurements from CT angiography (CTA) scans from the University of Maryland Medical Center, R Adams Cowley Shock Trauma Center database with eUnity software to analyze the position of the intermuscular septum between the anterior and lateral compartments of the leg. Participants $(n=100)$ were selected randomly from the years 2012-2019 who were admitted for a traumtic injury to the lower extremity and underwent a CTA. Patients ranged

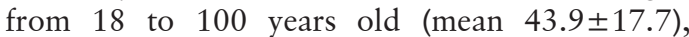
including 71 men and 29 women. Fasciotomy error rates, defined as how many patients needed to return to the operating room for repeat of a compartment decompression, were obtained from 

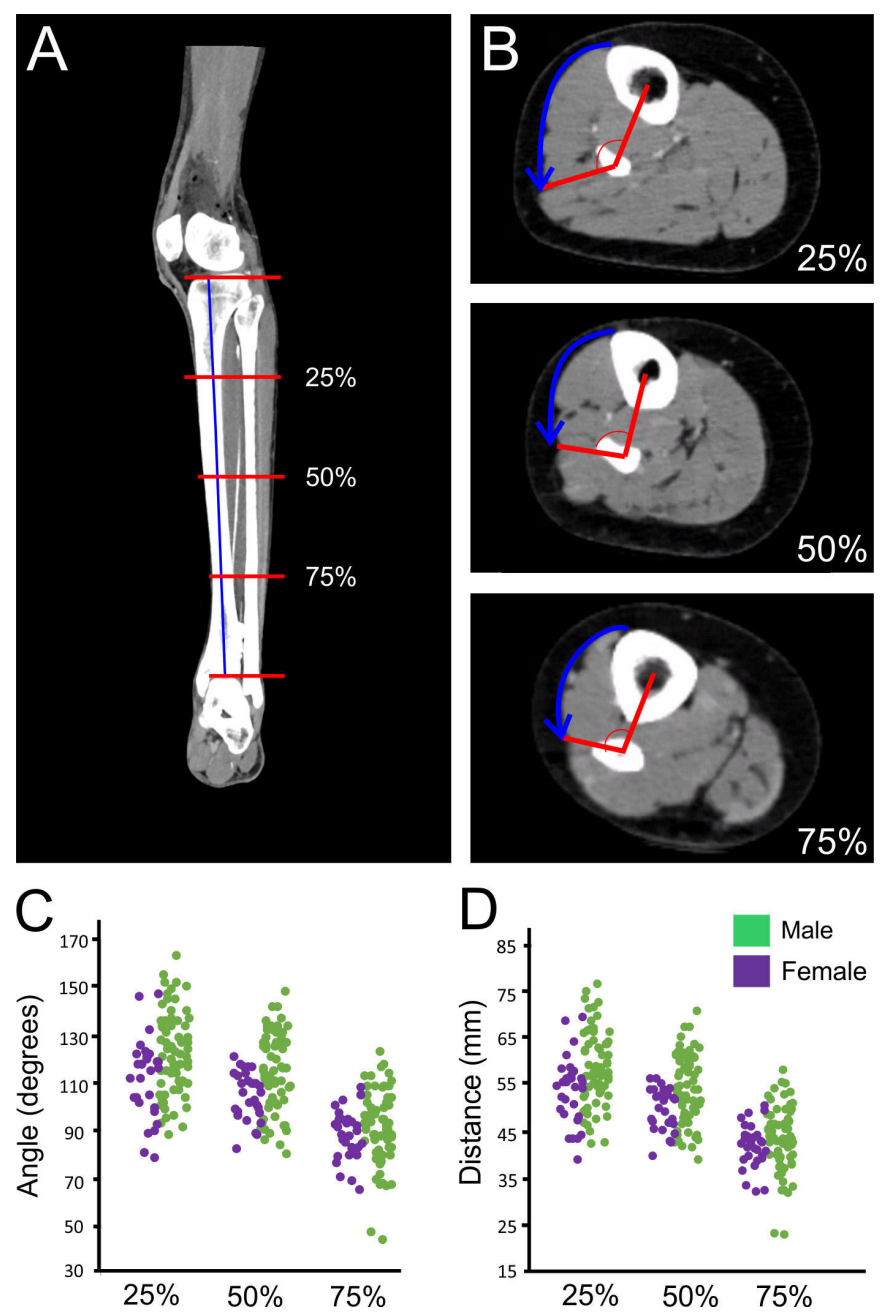

Figure 1 Diagrammatic representation of the measurement strategy. (A) Coronal CTA showing the $25 \%, 50 \%$, and $75 \%$ proximal to distal measurement points on the leg. (B) Tangential CTA of the patient (A) at $25 \%, 50 \%$ and $75 \%$ levels showing the angular measurements of the intermuscular septum between anterior and lateral compartments relative to the line between the fixed bony architecture of the tibia and fibula. (C) Scatter plot of individual patient measurements in degrees on the abscissa at the $25 \%, 50 \%$, and $75 \%$ of the leg length shown on the ordinate. (D) Scatter plot of individual patient circumferential crural fascia arc distance from the tibial edge in millimeters at proximal to distal positions at $25 \%, 50 \%$, and $75 \%$ of the leg length. $n=100$ patients.

electronic records from the University of Maryland Medical Center, R Adams Cowley Shock Trauma Center database of all patients receiving a leg fasciotomy between 2015 and 2021.

All measurements were performed using axial scans or reformatted sagittal scans and represent the mean value for the data set. The length of the leg was obtained from the tibia plateau to the distal end of the tibia shaft at the ankle joint. This study measured septal position relative to bony landmarks commonly used for surgical incision placement in the lower extremity fasciotomy procedure at three proximal to distal levels, 25\%, 50\%, and $75 \%$ of the distance from the tibial plateau to the ankle (figure $1 \mathrm{~A}, \mathrm{~B}$ ). At each of these levels, a line was drawn between the center of the tibia to the center of the fibula and a line from the center of the fibula to the skin over the junction of the intermuscular septum with the crural fascia. The angles between the tibia to the fibula line and the fibular to the septal junction were measured. A larger angle is a more posterior location for the intermuscular septum, whereas a shallower angle is a more anterior position.

As a second approach to assessing the position of the intermuscular septum between the anterior and lateral compartments, the arc sector circumferential length of the crural fascia from the tibia to the junction of the intermuscular septum with the crural fascia was measured. The arc segment circumferential length is independent of the position of the fibula, as the anterior measure is based solely on the tibia edge.

To assess the body habitus of leg tissues, we used the ratio of the circumference of the crural fascia to the circumference of the skin as a measure of leg subcutaneous tissue volume (ie, body habitus of the leg). Individuals who had a ratio close to one have a leg with limited subcutaneous tissue, whereas those who had lower fascia to skin ratio have larger amounts of subcutaneous tissue. To approximate muscular volume, the absolute circumference of the crural fascia was compared against the septal position angle.

To assess the effect of direct injury to the leg, patients were grouped by injury type into direct or indirect trauma to the leg. Injuries that were classified as direct were those that were the result of blunt or penetrating trauma to the leg, including gunshot wounds, fractures, etc. Indirect injuries were similar injuries occurring in the thigh or foot not directly involving the leg.

We conducted a review of the electronic record at the University of Maryland Medical Center database for all leg fasciotomies performed between 2015 and 2021, consisting of 151 patients. Statistical analysis was performed in Microsoft Excel 2019 using a t-test when comparing two variables, analysis of variance when comparing multiple covariants, and linear regression for the cross-correlation analysis.

\section{RESULTS}

\section{Error rates in performing lower extremity fasciotomy}

Of the 151 fasciotomies performed at our medical center, 11 contained one or more missed compartments that necessitated a return to the OR to complete the compartments (representing a $7.3 \%$ error rate). Anterior and posterior deep compartments were the most commonly missed. Seven out of the 11 patients required reoperation to complete an anterior compartment decompression. There was an additional case of a missed lateral compartment, which conceivably also could have been related to the lateral/anterior septal position. Five of 11 reoperations involved the posterior deep compartments and only 1 involved the posterior superficial compartment.

\section{Position of the intermuscular septum between anterior and lateral compartments}

The average septum positional angle was $119.2^{\circ} \pm 17.5^{\circ}$ (range 163.0 to 78.9 , male/female pooled; figure $1 \mathrm{C}, \mathrm{D})$ at the $25 \%$ distal level, which is typically the most muscular part of the leg and $112.5^{\circ} \pm 15.2^{\circ}$ (range 148 to 80.6 ) at $50 \%$ distance. Distally at the $75 \%$ location, the septum was significantly more anterior with an angle of $90.5^{\circ} \pm 13.8^{\circ}$ (range 123 to 45.1 ). The positional angle was not significantly different between men and women (figure 1C,D).

As with angle measures previously mentioned, the arc segment circumferential length mean was $5.7 \mathrm{~cm} \pm 0.81 \mathrm{~cm}$ (range $7.68 \mathrm{~cm}$ to $3.89 \mathrm{~cm}$ ) at the $25 \%$ distal level, 5.34 $\mathrm{cm} \pm 0.71 \mathrm{~cm}$ (range $7.1 \mathrm{~cm}$ to $3.90 \mathrm{~cm}$ ) at the $50 \%$ distal level and $4.23 \mathrm{~cm} \pm 0.63 \mathrm{~cm}$ (range $5.83 \mathrm{~cm}$ to $2.29 \mathrm{~cm}$ ) at the $75 \%$ 
A

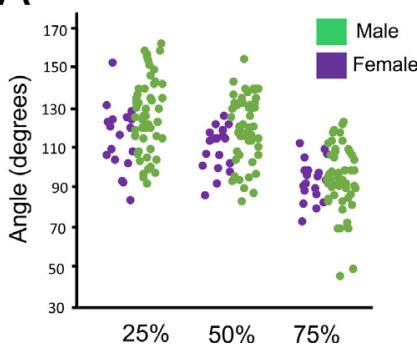

B

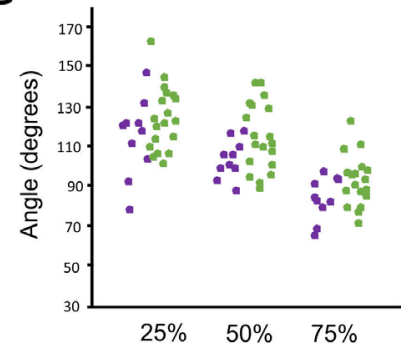

Figure 2 Comparison of septal position in patients with direct and indirect lower limb injury. Measurements are from proximal to distal positions of $25 \%, 50 \%$, and $75 \%$ of the leg length. Angular measurements are of the position of the intermuscular septum between anterior and lateral compartments relative to the line between the fixed bony architecture of the tibia and fibula. (A) Scatter plot of septal angle in individuals who had direct injuries to the leg, $n=71$ patients. (B) Scatter plot of the septal angle in individuals who had indirect leg injuries, $\mathrm{n}=29$.

distal level. There were no significant differences between male and female patients. As both angle and arc length were consistent, thus for the correlations below only angle measures are described.

\section{Does injury type affect intermuscular septal location?}

There were no statistically significant differences in septal position between direct and indirect injuries at the 25\%, 50\%, or $75 \%$ position distally on the leg ( $\mathrm{p}$ values of $0.47,0.29$, and 0.23 , respectively). For direct injuries, the average angle made between the bony architecture and the intermuscular septum at the $25 \%$ distal level was $118.4^{\circ} \pm 17.4^{\circ}$ (range 81.1 to 155.0 , figure $2 \mathrm{~A}$ ), whereas for indirect injuries the angle was $121.2^{\circ} \pm 17.1^{\circ}$ (range 78.9 to 163.0 , figure $2 \mathrm{~B}$ ). At the $50 \%$ distal level, direct injury angles were $113.0^{\circ} \pm 15.0^{\circ}$ (range 80.6 to 148.0 , figure $2 \mathrm{~A}$ ) and indirect injury angles were $111.1^{\circ} \pm 15.3^{\circ}$ (range of $88.4^{\circ}$ to $142.0^{\circ}$, figure $2 \mathrm{~B}$ ). At the $75 \%$ distal level, the angle for direct injuries was $90.5^{\circ} \pm 14.2^{\circ}$ (range 45.1 to 118.0 , figure $2 \mathrm{~A}$ ) and that for indirect injuries was $90.7^{\circ} \pm 12.6^{\circ}$ (range 65.9 to 123.0 , figure $2 \mathrm{~B}$ ).

A
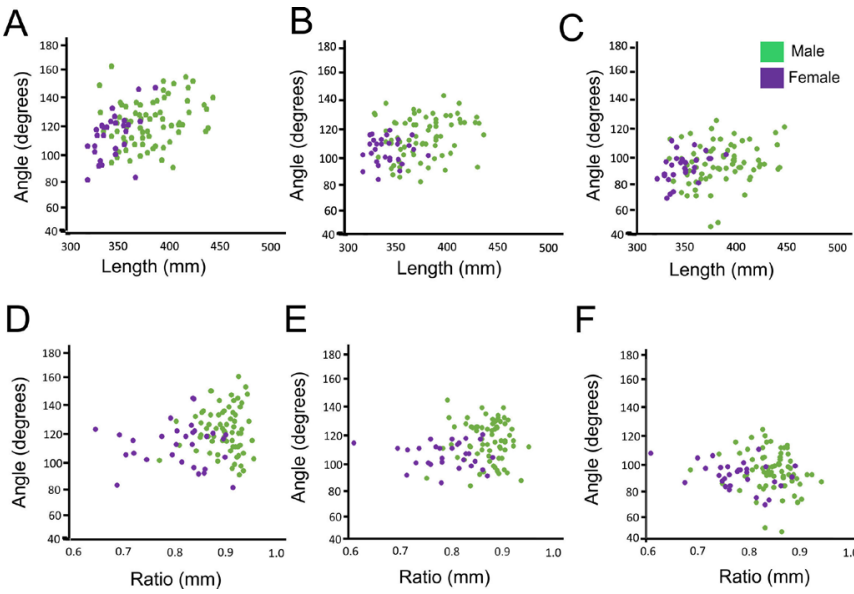

F

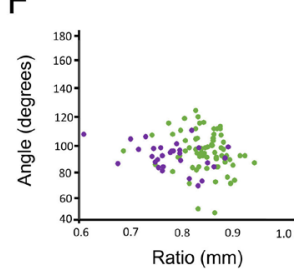

Figure 3 Comparison of septal position versus leg length $(A-C)$ and versus body habitus (D-F) at proximal to distal positions of $25 \%(A, D)$, $50 \%(B, E)$, and $75 \%$ of the leg length $(C, F)$.

Does leg length or body habitus predict septal position?

At all three levels, there was no clinically relevant correlation of septal angle compared with leg length (figure 3A-C). This study also yielded no clinically useful relationship between the body habitus metric and septal angle (figure 3D-F). There was no significant correlation between body habitus and leg length, yielding results of $\mathrm{r}=0.38,0.33$, and 0.25 at positions $25 \%$, $50 \%$, and $75 \%$ along the leg for leg length and $r=0.24,0.27$, and 0.20 , respectively, for body habitus. Female patients exhibited shorter leg length consistent with average height difference between male and female general populous and slightly higher body habitus (lower ratio of skin to crural fascia) consistent with the approximately $20 \%$ higher body fat content of female to male in the populous (Centers for Disease Control National Health and Nutrition Examination 1999-2004). As with leg length and body habitus, there were no clinically useful relationships between the volume of muscular compartments and the expected position of the intermuscular septum (not shown).

\section{DISCUSSION}

This study of lower extremity anatomy based on CT imaging revealed that the position of the intermuscular septum between the anterior and lateral compartments were widely variable in position between individuals relative to bony landmarks commonly used for surgical incision placement. Thus, the proposition that the intermuscular septum is always going to be directly deep to an incision one finger anterior to the fibular in all individuals is incorrect. The data further show that a fixed incision spacing along the length of the leg, for example, one finger in front of the fibula, would be slightly tangential to the line formed by the intermuscular septum distal in the leg.

In any trauma surgery, the specifics of the patient injury and associated potential disruption of the anatomy are always a prime factor in surgical planning. In the case of leg fasciotomy, this could include disruptions of fascial compartments from tibial or fibular compound fracturing or other major injury altering landmarks. Thus, a 'standard' incision, although reducing error in compartment identification, should always be modified as warranted to best meet the specifics of the individual patient's injuries.

In the two-incision four-compartment leg fasciotomy, the lateral compartment is commonly decompressed, but the anterior compartment is frequently missed. ${ }^{5}$ The need to correctly identify the intermuscular septum between the anterior and lateral compartments is critical to adequate decompression of these compartments. We hypothesized that a contributing factor to the error rate in anterior compartment decompression may be a surgeon misidentifying the septum between the lateral and posterior superficial compartments as the septum between anterior and lateral compartments. This would result in a failure to decompress the anterior compartment and 'double' decompressing the superficial posterior compartment from both sides of the leg.

Why could this erroneous septum be 'found' frequently enough to explain the error rates in Mackenzie $e t \mathrm{al}^{7}$ of approximately $10 \%$ and the error rates observed in our retrospective chart review in this study of $7.3 \%$ necessitating return to the operating room for one or missed compartments (64\% of which involved the anterior compartment)? The data in this study shows that intermuscular septum position varies considerably between individuals. In a patient with a shallow septum angular position, the intermuscular septum is more anterior and closer to the tibia than expected. From an initial incision, the natural 

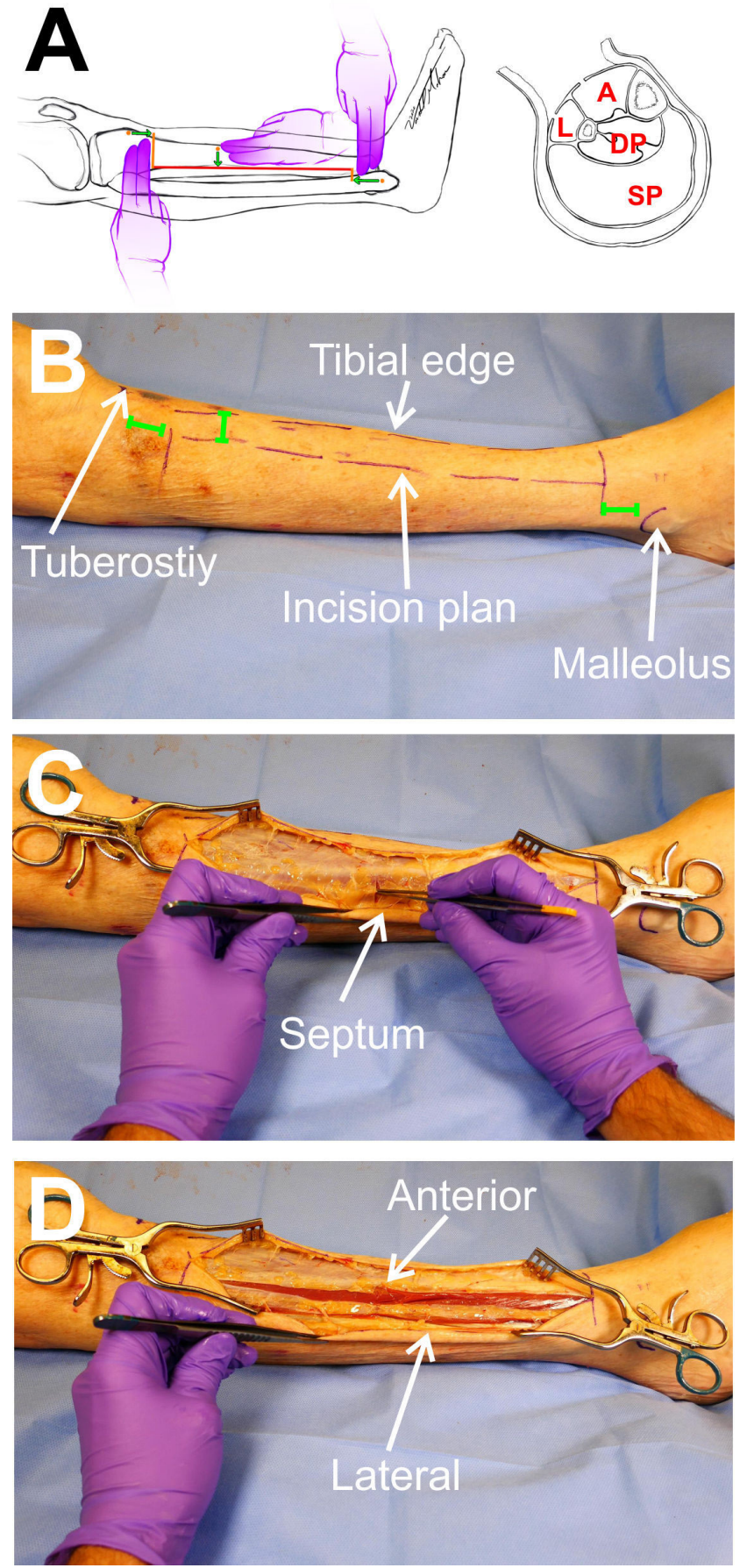

Figure 4 Diagrammatic and cadaveric demonstration of the new incision placement in performance of the lower extremity fasciotomy. (A) Schematic showing the incision placement of two fingers distal to the tibial tuberosity, two fingers proximal to the lateral malleolus, and two fingers posterior to the tibia. Side panel represents a cross section for the leg compartments ( $A, L, S P$, and SD) with the superficial and deep fascia exposed for the anterior and lateral decompression. (B) Landmarks and incision placement using the tibial edge as the primary bony landmark. The planned incision is placed two finger breadths (green marks) posterior to the tibia and extending from two fingerbreadths distal to the tibial tuberosity and two finger breaths proximal of the lateral malleolus. (C) H-shaped incision spanning the anterior and lateral compartments across the intermuscular septum. (D) Decompressed anterior and lateral compartments from incisions in the crural fascia. Artwork by Vondel Mahon. A, anterior; L, lateral; SP, superficial posterior, and SD superficial deep. tendency of the surgeon is likely progression posteriorly rather than 'up and under' the skin in an anterior direction. This could result in the surgeon first finding the septum between the lateral and posterior superficial compartments just posterior to their incision and drawing an inaccurate conclusion to the septum identity. Furthermore, the septum shifts relatively more anteriorly as it progresses to the distal leg yielding a changing septum position relative to the bony landmarks. This could lead to the crural fascia decompressive opening being incomplete if the surgeon does not compensate to parallel the slight anterior to posterior line of the septum when opening anterior and lateral compartments.

Since septum position varies from patient to patient, making the initial incision slightly more anterior may result in enhanced view and easier access for surgeons to find the correct intermuscular septum. Our conjecture is that this may lead to reduced error of a missed anterior compartment decompression. Thus, we propose that the initial incision placement should be moved slightly more anterior and use the tibial spine as the primary landmark rather than the fibula. The course of the fibula is difficult to palpate precisely in a non-swollen leg, and that difficulty increases when there is direct trauma to the leg or tissue edema associated with swollen leg compartments as a patient develops compartment syndrome. The superficial position of the anterior tibial surface, which lacks musculature, is more readily palpable in both swollen and non-swollen legs.

We propose guidelines for the initial incision should be two finger breadths posterior the tibial spine (figure 4). The length of the incision would remain the currently recommended two fingerbreadths distally to the tibial tuberosity and two fingerbreadths proximally to the lateral malleolus. This revised incision placement ensures that the surgeon is more conservatively positioned relative to the variations observed in the intermuscular septum position and gives the surgeon easier working room to progress from anterior to posterior to localize the septum. This also makes all landmark references medial and lateral in the fasciotomy to be two fingers and allows for a simple recall mnemonic of 'two fingers from the tibia' for all landmarks needed. This proposed change to the procedure landmarks could lead to higher success rate by limiting identification error and the need for performing a fasciotomy a second time to correct the error.

Acknowledgements We thank the Department of Diagnostic Radiology and Nuclear Medicine for the use of their database and the Department of Defense (W81XWH-17-2-0011) for funding this project.

Contributors LA: investigation, writing (original draft) and methodology; KF: methodology and conceptualization; KS: formal analysis and methodology; ST and ACP: supervision, writing (reviewing and editing) and conceptualization.

Funding Funding for this project was provided by the Department of Defense (award number W81XWH-17-2-0011, log number BA150808).

Competing interests $\mathrm{MB}$ is a consultant for grants held by the senior authors. Patient consent for publication Not required.

Ethics approval The work was conducted under protocols approved by the University of Maryland School of Medicine Institutional Review Board.

Provenance and peer review Not commissioned; externally peer reviewed.

Data availability statement All data relevant to the study are included in the article or uploaded as supplementary information. Data are available upon request and are available from Dr Adam Puche, apuche@som.umaryland.edu.

Open access This is an open access article distributed in accordance with the Creative Commons Attribution Non Commercial (CC BY-NC 4.0) license, which permits others to distribute, remix, adapt, build upon this work non-commercially, 
and license their derivative works on different terms, provided the original work is properly cited, appropriate credit is given, any changes made indicated, and the use is non-commercial. See: http://creativecommons.org/licenses/by-nc/4.0/.

\section{ORCID iDs}

Lorreen Agandi http://orcid.org/0000-0001-7378-5062

Samuel Tisherman http://orcid.org/0000-0003-3810-3729

\section{REFERENCES}

1 Henderson AP. Compartment syndrome: medical causes, symptoms and surgical outcomes. nova biomedical. Hauppauge, NY, 2015.

2 Bowyer MW. Lower extremity fasciotomy: indications and technique. Curr Trauma Rep 2015:1:35-44.

3 Singh K, Bible JE, Mir HR. Single and Dual-Incision fasciotomy of the lower leg. JBJS Essent Surg Tech 2015;5:e25.
4 American College of Surgeons Committee on Trauma. ASSET (advanced surgical skills for exposure in trauma) exposure techniques when time matters. Chicago, IL: American College of Surgeons, 2010.

5 Mackenzie CF, Bowyer MW, Henry S, Tisherman SA, Puche A, Chen H, Shalin V, Pugh K, Garofalo E, Shackelford SA, et al. Cadaver-Based Trauma Procedural Skills Training: Skills Retention 30 Months after Training among Practicing Surgeons in Comparison to Experts or More Recently Trained Residents. J Am Coll Surg 2018;227:270-9.

6 Large TM, Agel J, Holtzman DJ, Benirschke SK, Krieg JC. Interobserver variability in the measurement of lower leg compartment pressures. J Orthop Trauma 2015;29:316-21.

7 Mackenzie CF, Shackelford SA, Tisherman SA, Yang S, Puche A, Elster EA, Bowyer $M W$, Retention and Assessment of Surgical Performance Group of Investigators. Critical errors in infrequently performed trauma procedures after training. Surgery 2019;166:835-43. 